\title{
Development of Low Cost Miniature USB Based Data Acquisition System for Biomedical Instrumentation
}

\author{
Abdullah Al Amin*, K Siddique-e Rabbani \\ Department of Biomedical Physics and Technology, University of Dhaka \\ Email: a.amin@bmpt.du.ac.bd, rabbani@du.ac.bd \\ * Corresponding author
}

[Received: 15 March, 2018, Accepted: 19 September, 2018]

\begin{abstract}
In biomedical instrumentation, computer based data acquisition system is required for recording of physiological parameters and bioelectric signals, which allows signal processing, display, analysis and storage in digital media. However, Most of the commercially available PC based Data Acquisition systems are of very high cost and requires specific commercial software, again at a very high cost. Moreover, if the data is not stored in raw binary or known format, it is not possible for the user to use the data in other system or software of their own choice. Therefore, a low cost, simple and open source PC based data acquisition system for biomedical application would be very useful for biomedical instrument developers and researchers in the low resource countries. In this work, we present such development of data acquisition system. The developed system utilizes an 8-bit ordinary low cost microcontroller and some electronic circuit component to develop the data acquisition system and implementation of USB 1.1 (Universal Serial Bus) interface to PC. The onboard 10-bit ADC of the microcontroller was used for analog data sampling. Two sampling and data transfer mode is implemented, (i) Continuous mode with low sampling rate (800 sample/sec) and practically real time plotting and (ii) Batch mode, with high sampling rate $(76.9 \mathrm{k} \mathrm{sample/sec})$ but with batch type plotting. To evaluate the system, PC side GUI (Graphical User Interface) software was also developed. The GUI of system shows that a test sinusoidal signal is reproduced very nicely without any amplitude and phase distortion within the frequency band of 1 to $10 \mathrm{KHz}$. The system is suitable for low frequency bioelectric signals like ECG, EEG etc. and as well as high frequency signal like EMG, NCV etc. The system is low cost, miniature, simple, and efficient and being used in several indigenously developed medical devices like ECG, EMG, NCV and FIM [Rabbani et al, 1999] at the authors' department with excellent satisfactory results.
\end{abstract}

Keywords: USB Interface, ADC, Data Sampling, Data Acquisition. 


\section{INTRODUCTION}

Computerized medical equipment are becoming more and more popular because of ease of medical data pre/post processing and analysis, storage and printing, usability in telemedicine etc. It reduces hardware count and complexity of medical device, moreover multiple devices can use one PC, thus making the total system more economical, easy to maintain and repair. Currently, wide availability of PC (Personal Computer) all over the world has made this type of system more feasible.

Usually PC provides several interface ports for external devices; Parallel, Serial, Ethernet, FireWare, USB (Universal Serial Bus) etc. Among these USB has become more widespread and has almost eliminated the use of other interfaces at the user end.

Parallel and Serial ports are becoming obsolete as these are bulky, designed for short distance, and require separate external power supply at the device end. Moreover, the latter has comparatively lower data rate then the other PC interfaces. These two ports are currently rare as modern PC or motherboard manufacturers are omitting these from their design. Ethernet is commonly used for network printers and some medical equipment for its very fast data rate and electrical isolation feature but the drawbacks are high cost and requirement of external power for devices, which in turn needs isolation again for medical devices. FireWire and USB interfaces are superior to others by physical dimension, speed and the ability to source power to the device. The only drawback of these interfaces for use in medical equipment is that the signal and power are not isolated. FireWare is more expensive than USB because of its patent rights and royalty per device at user end, thus leaving USB as most adopted interface.

USB version 1.0 was first introduced by USB-IF (USB Implementers Forum) in 1996 [USBIF, 2014], it was intended to replace multiple different ports for different devices and provide one single interface for all PC based equipment. It has gone through improvements over time and the most recent version is 3.0. USB has an open specification, however to implement it at device end, manufacturers have to purchase Vendor ID (VID) from USB-IF, which is one-time payment for lifetime, required for regulation and maintenance of USB devices. VID needs to be unique as host application assigns device driver by recognizing VID and PID (Product ID) pair. The PID is a 16 bit field of manufacturer's choice allowing up to 65535 different devices for each purchased 
VID. Additionally, if the manufacturers wish to use the USB logo, they have to become a registered user end member of USB-IF by an annual fee. USB has several standard specifications: version 1.0 and 1.1 have low speed (1.5 Mb/s), version 2.0 supports low, full (12 $\mathrm{Mb} / \mathrm{s}$ ) and high speed $(480 \mathrm{Mb} / \mathrm{s})$, while version 3.0 is Super Speed (5 Gb/s) with backward compatibility of all versions. This current work implements USB version 1.1 low speed PC interface for data acquisition. The system is intended for medical devices that measures bioelectric signal or physiological parameters that are converted into electrical quantity.

In order to transfer the analog bioelectric signal to PC through USB interface, it must be sampled in time and each value is converted to digital value using an analog to digital converter (ADC). Usually periodic samples with equal time intervals are acquired and these represent the series of digital formation of the time varying analog signal. The total system is then called data acquisition system. Since PC side of the system is usually connected to mains as power line, special patient safety consideration is necessary for medical equipment. The bioelectric signals from human subject or patient and associated circuitry for signal conditioning (amplification, filtering etc.) at the patient end must be electrically isolated form the circuitry associated with PC interface. The power source for the patient side circuitry needs to be isolated as well.

This current work is a local adoption of USB interface for medical data acquisition which is a part of the effort to overcome the technological and economic disparity between the First and Third world countries through indigenous development of technology. It was interesting to find out that even after two decades of introduction of USB with open specification, no work reference or people with experience with USB implementation in both software and firmware were found in a developing country like Bangladesh. It is an indication of the technological disadvantage of developing countries.

This paper presents details of the development of a medical grade USB data acquisition system including power and signal isolation, analog to digital conversion at precise time intervals for sampling, and PC interface for synchronous data transfer through implementation of USB protocol at both device hardware and PC side software. 


\section{METHODS}

The hardware system is designed around a low cost 8-bit Atmel AVR ATmega8 [Atmel, 2014] microcontroller with integrated analog to digital converter (ADC). The associated circuitry needs only an isolation amplifier, which is used to achieve electrical isolation between the mains ac and bioelectrical signal source (human body) when the system is connected to PC (mains connected). This isolation is required for user safety and to reduce noise from the mains line by increasing Isolation Mode Rejection Ratio (IMRR) [Van Rijn et al., 1991]. Some optocouplers are used to isolate digital I/O's for the same reason and a few passive components are used. This design reduced the cost and complexity very much.

With reference to Figure 1, the analog bioelectric signal from the human body is first conditioned and transferred to the non-isolated PC side through the analogue isolation amplifier mentioned above. Then the signal is conditioned to meet the ADC input requirement. The signal is then converted to digital values using the on-board 10-bit ADC of the microcontroller. The digital data is then transferred to the PC through its USB port. Necessary USB interface [USBIF, 2014] was developed for this purpose, based on the same microcontroller.

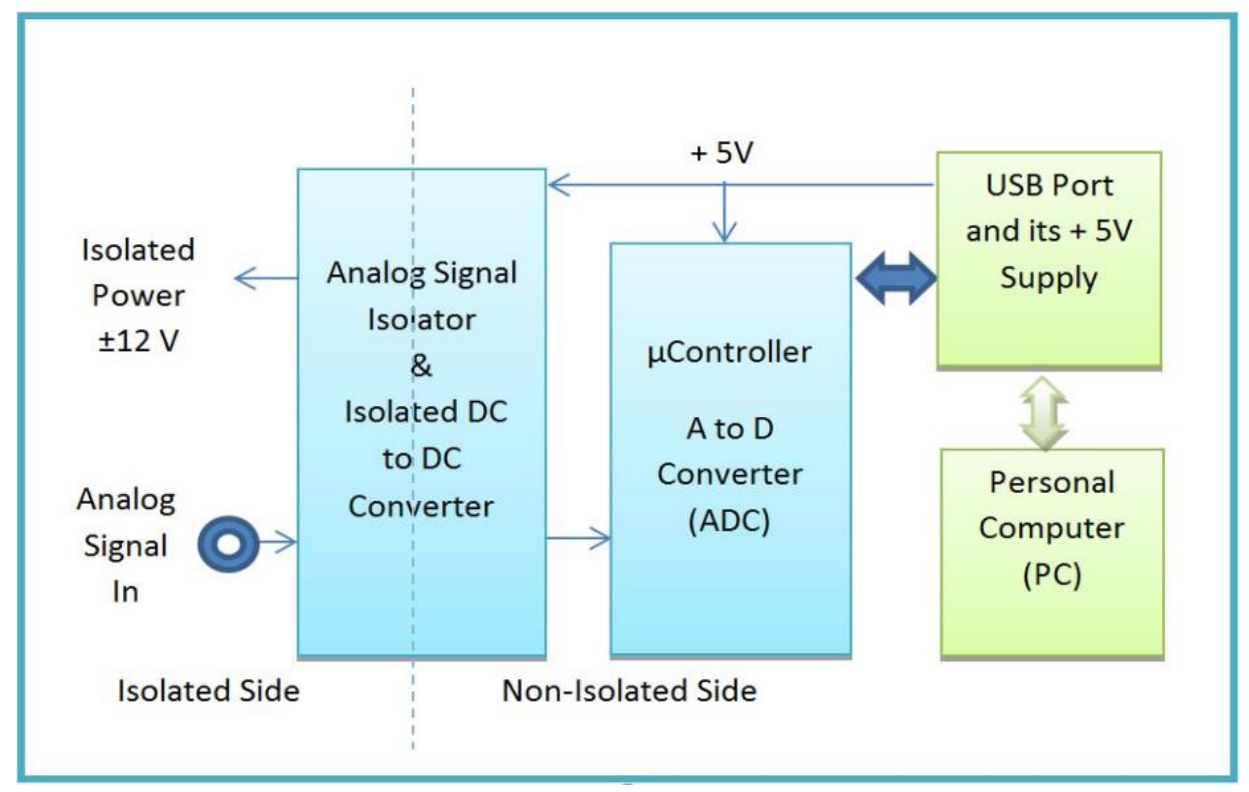

Fig. 1: Block Diagram of Data Acquisition System 
For powering the isolated circuitry in the isolated side, a medical grade isolated dc to dc converter is used; it uses the non-isolated +5 volt supply available from the USB port of the PC, and produces \pm 12 volt split power at the isolated side. Therefore, no external battery or power supply is needed when the system is connected to PC. Figure 2 shows the circuit diagram of the Data Acquisition system.

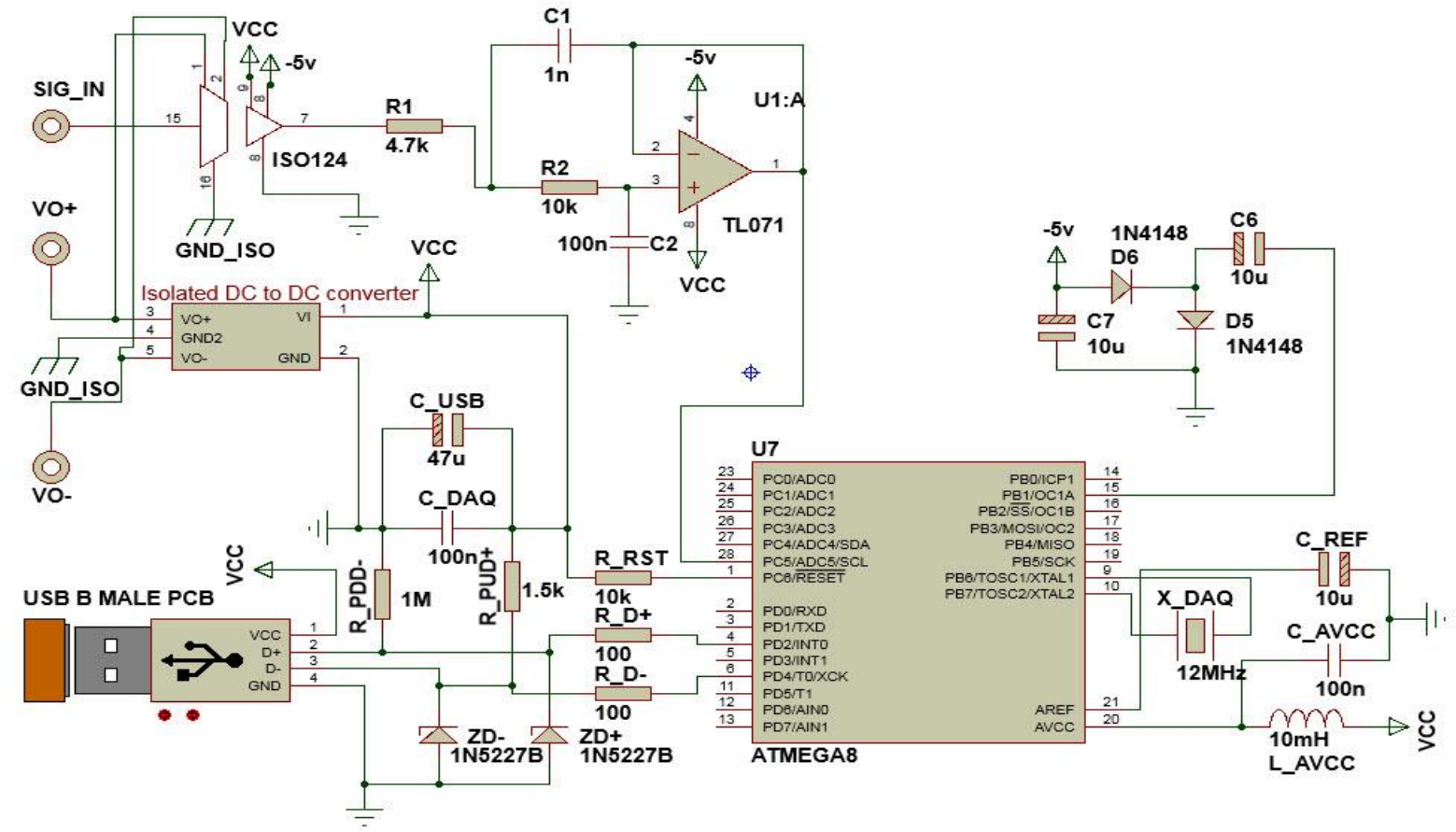

Fig. 2: Circuit Diagram of Data Acquisition System

The microcontroller has 10 bit successive approximation ADC module, it has two selectable internal references $5 \mathrm{~V}$ and $2.56 \mathrm{~V}$. The $2.56 \mathrm{~V}$ is used for this device for stability and higher voltage resolution of the bioelectric signal. After digitally converting the signal most significant 8 bits are used as the least significant two bits are more prone to noise. The 8 bits are kept also for the reason that the byte wise data storage and transfer is more efficient. The $2.56 \mathrm{~V}$ reference of the ADC produces the resolution of $10 \mathrm{mV}$ per bit for 8 bit conversion. 
The device operates in two modes,

(i) Continuous conversion and data transfer: The sampling frequency is $800 \mathrm{~Hz}$, it is selected to match the USB 1.1 periodic 'Interrupt In' timing and data transfer rate. This sampling rate is suitable for low frequency signals like ECG, EEG etc.

(ii) Batch transfer: The data can be sampled very fast, i.e. $13 \mu$ s per conversion (recommended minimum conversion time of the ADC module of the microcontroller) provides maximum of $76.9 \mathrm{KHz}$ of sampling rate. The data is stored in a large SRAM space of 800 bytes in the microcontroller and then transferred periodically by USB 'Control In' transfer.

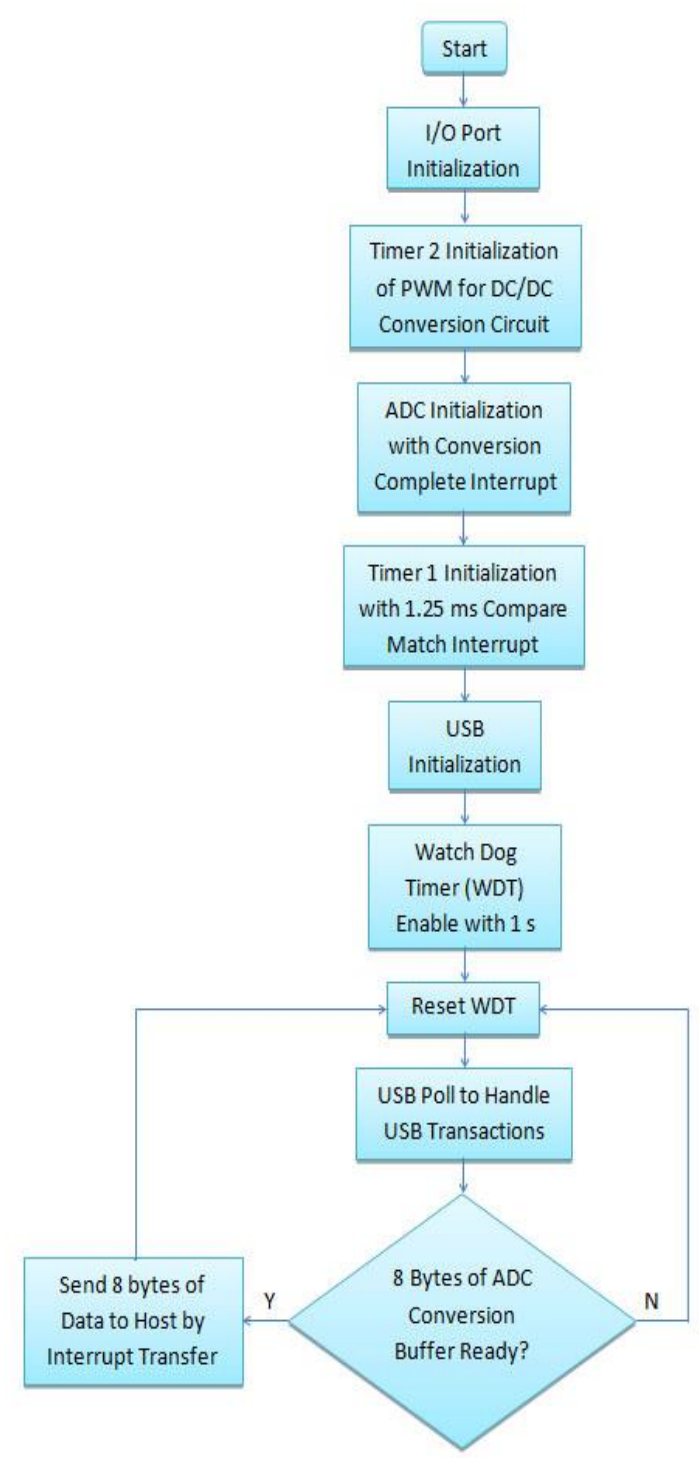

Fig. 3: Continuous Conversion Flowchart 
These modes are described in details below.

(i) Continuous conversion and transfer mode:

The Continuous conversion data is transferred by 'Interrupt In transfer' for graphical display and storage by the developed software. By specification this 'Interrupt In transfer' type has maximum of 8 bytes transfer every $10 \mathrm{~ms}$. The USB host controller generates this timing and it is adopted as que for starting ADC conversion. So, at every $10 \mathrm{~ms}$ there are 8 bytes of data conversion takes place at equal interval of $1.25 \mathrm{~ms}(10 / 8 \mathrm{~ms})$, resulting sample rate of $800 \mathrm{~Hz}$. The $1.25 \mathrm{~ms}$ interval for analog data conversion is precisely generated using the 16 bit 'Timer 1' module of the microcontroller; this timing generates an interrupt to start ADC conversion at every $1.25 \mathrm{~ms}$. The sampling and data transfer is done simultaneously and these are well synchronized. Thus from sampling to display has latency of $10 \mathrm{~ms}$, which can be regarded as real time. The Flowchart of the Continuous mode firmware is shown in Figure 3.

\section{ii) Batch Transfer:}

The Batch Transfer uses both 'Interrupt In' and 'Control In' transfer of the USB. The Flowchart of the Batch Transfer mode firmware is shown in Figure 4.

The 'Interrupt In' transfer cannot provide data transfer rate more than 800 bytes/sec (max. 8 bytes per $10 \mathrm{~ms}$ ) but has guaranteed time of $10 \mathrm{~ms}$ interval of each transfer. This timing is used as synchronization with PC software and start of conversion Que. The 'Control In' transfer can transfer large amount of data (maximum of $1.5 \mathrm{Mbit} / \mathrm{sec}$, depending on the bus bandwidth availability) but its transfer timing is not guaranteed. This transfer is primarily specified for transferring device descriptors and other host control over USB, but it is not forbidden to use 'Control In' for other data transfers in the USB specification. The ADC sampling interval is controlled in similar manner like the Continuous mode but now this interval can be as low as $13 \mu \mathrm{s}$. The software polls the device to acknowledge 800 bytes (this is the current microcontroller's maximum SRAM free space after other use) of batch data conversion accomplishment, every $10 \mathrm{~ms}$ through 'Interrupt In' transfer. If the data is available, the software initiates a 'Control In' transfer for the 800 bytes data. After 
transferring the batch data to PC, the 'Interrupt In' polling is used to initiate converting another batch of data.

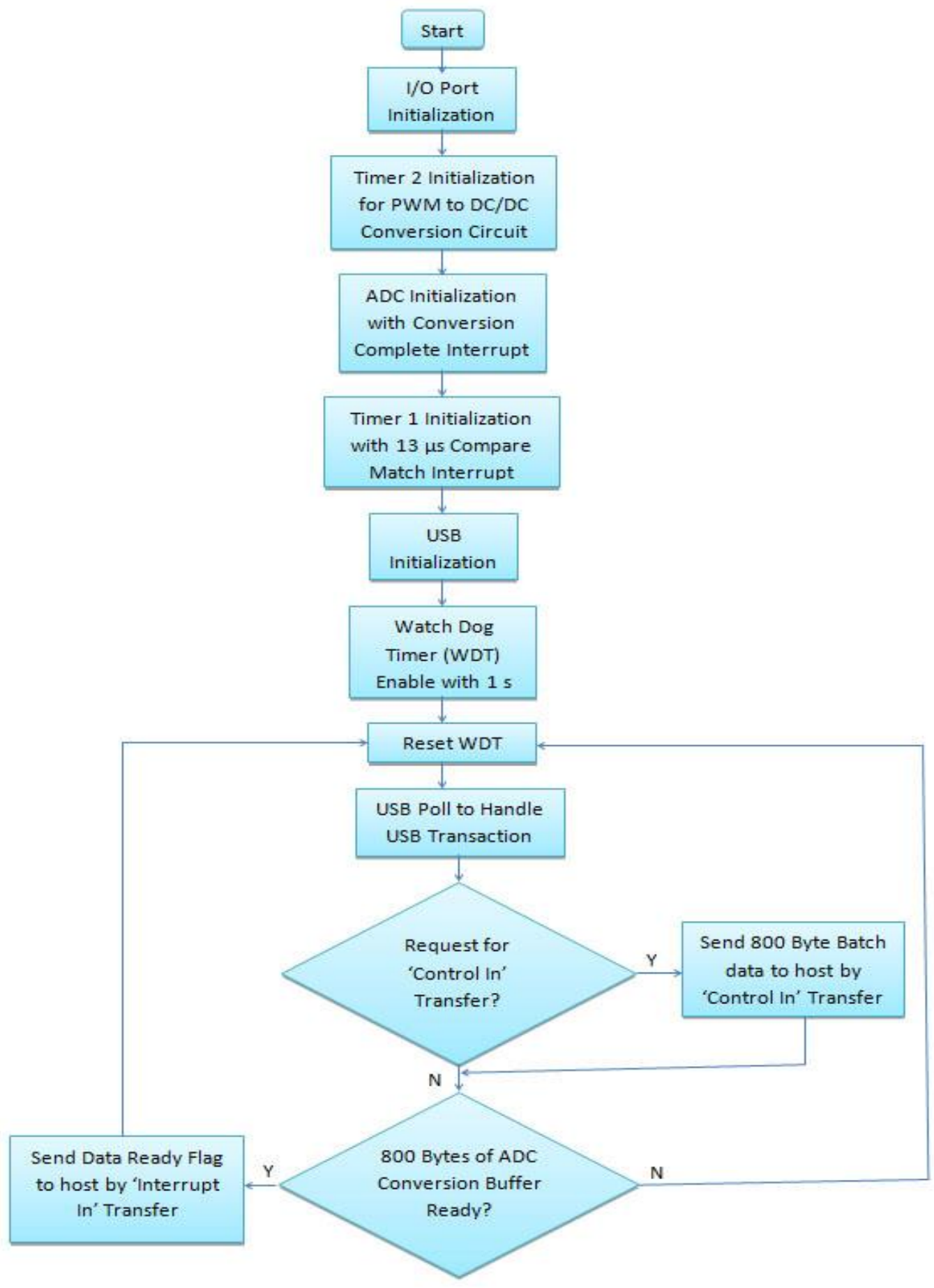

Fig. 4: Batch Mode Conversion Flowchart 
Since the microcontroller is low cost and have no hardware USB stack, the USB library called V-USB [Objective D., 2011] of the firmware was used for implementation of USB 1.1 low speed standard protocol. The firmware for the microcontroller was developed using free GNU C compiler [WinAVR, 2014] with AVR Studio IDE [Atmel, 2014]. The software is a graphical user interface which allows viewing the bioelectrical signal in selectable different time scale and can store data in raw binary format. It was developed using 'JAVA' platform considering its operating system (OS) independent feature. The open source and free development environment (IDE) 'Netbeans' [Oracle, 2014] was used for development of this software.

\section{RESULTS AND OBSERVATIONS}

A sinusoidal wave of $80 \mathrm{~Hz}$ was fed from a signal generator to the data acquisition system and resulting display on a PC produced by the developed software is shown in Figure 5.

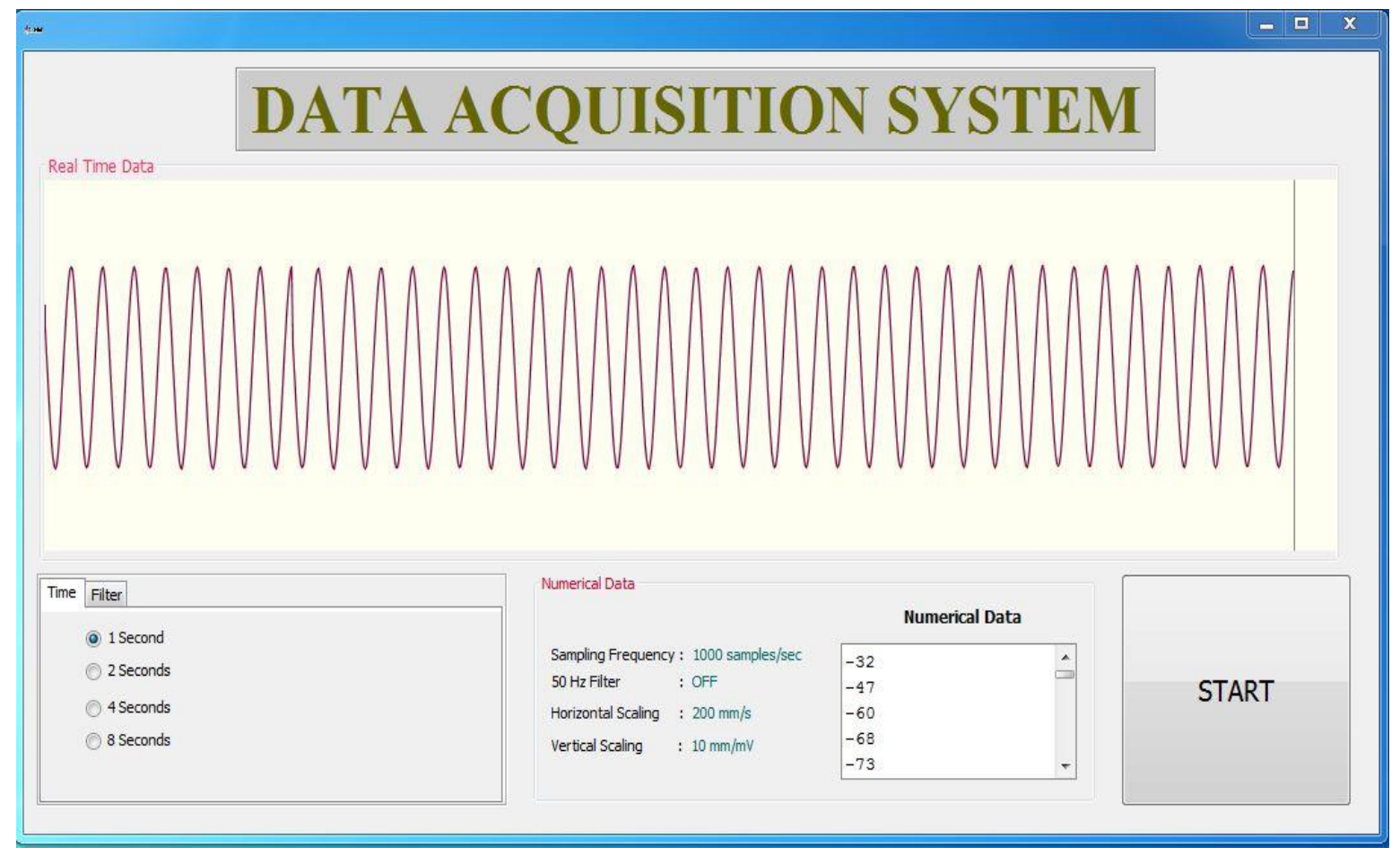

Fig. 5: GUI software of Data Acquisition System 
The GUI of the software developed for this system has a main window that displays the graphical form of the data. It also has selectable options for the time window from 1 to 8 seconds. The software implements an 8th order IIR digital notch filter with stop band cantered at $50 \mathrm{~Hz}$. This notch filter can be selected to turn on and off. Another display shows the configuration statuses, like data sampling rate, vertical and horizontal scaling etc. There is also a window which displays the numerical values of the digitized data.

It may be observed that the system reproduces the signal very nicely in PC. Further measurement at different frequencies showed that for Continuous conversion mode and Batch mode, aliasing effect was seen after $150 \mathrm{~Hz}$ and at $12 \mathrm{KHz}$ respectively. The system showed no significant phase or amplitude change up to $100 \mathrm{~Hz}$ and $10 \mathrm{KHz}$ for Continuous conversion and Batch mode respectively.

\section{DISCUSSION}

Though, USB specifies medical device class, the HID type was chosen because most of the current PC operating system provides driver for this class, thus unnecessary complexity of PC side driver development could be avoided. In continuous conversion and transfer mode, the sampling and data transfer are done simultaneously and are well synchronized. From sampling to display the system has a latency of approximately $10 \mathrm{~ms}$, which can be regarded as real time. This transfer mode is suitable for low frequency signals like ECG, EEG etc. The Batch transfer mode provides higher sample rate and it is suitable for high frequency bioelectric signals like EMG, NCV etc. The system is small with low component count and therefore cost effective, making it suitable for standalone systems as well.

Most of the bioelectrical signals occur within the band width of 1 to $10 \mathrm{KHz}$; therefore this system satisfies the biomedical instrumentation requirements. The only limitation of the system is the ADC resolution, which is comparatively low, but it is acceptable for most of the application stated before. It is possible to use several ADC channel for data acquisition, since the microcontroller has 6 ADC channels and minimum conversion time of $13 \mu$ s which would be negligible time laps between multiple conversions. If it is required to have higher resolution of 
ADC, larger SRAM and more ADC channels, different microcontroller can be used with the same working procedure since the firmware is written in $\mathrm{C}$ language.

The system is being used in ECG, EMG, NCV and FIM [Focused Impedance Measurement system, developed by Rabbani et al, 1999] with excellent satisfactory results.

\section{Acknowledgement}

One of the authors (A A Amin) wishes to gratefully acknowledge the International Science Programme of Uppsala University, Sweden for providing scholarship during his $\mathrm{PhD}$ work.

\section{REFERENCES}

Atmel Corporation (2014). Retrieved March 10, 2014, from: <http://www.atmel.com>

Objective Development Gmbh (2011). Virtual USB port for AVR microcontrollers. Retrieved August 15, 2011, From: http://www.obdev.at/products/vusb/index.html

Oracle Corporation (2014). NetBeans IDE, Retrieved March 10, 2014, From: 〈www.netbeans.org>

Rabbani, K.S., Sarker, M., Akond, M.H.R. and Akter, T., 1999. Focused impedance measurement (FIM): a new technique with improved zone localization. Annals of the New York Academy of Sciences, 873(1), pp.408-420.

USBIF (2014). Universal Serial Bus Implementation Forum. Retrieved Jan 14, 2014, from:

$<$ http://www.usb.org>

Van Rijn, A.M., Peper, A. and Grimbergen, C.A., 1991. The isolation mode rejection ratio, in bioelectric amplifiers. IEEE Transactions on Biomedical Engineering, 38(11), pp.1154-1157.

WinAVR (2014). Retrieved March 10, 2014, From:

http://www.webring.org/l/rd?ring=avr;id=59; url=http\%3A\%2F\%2Fwinavr\%2Esourceforge\%2Enet\%2F 